\title{
Positron emission tomography scan can be a reassuring tool to treat difficult cases of infective endocarditis
}

\author{
Tsuneaki Kenzaka, MD, ${ }^{\text {a }}$ Miho Shimoshikiryo, MD, ${ }^{\mathrm{b}}$ Akihito Kitao, MD, \\ Kazuomi Kario, $M D,{ }^{c}$ and Masayoshi Hashimoto, $M^{d}$
}

Introduction. The diagnosis of infective endocarditis is typically based on clinical presentation, positive blood cultures, and echocardiography findings. In particular, echocardiography is central to the diagnosis and management of patients with infective endocarditis. ${ }^{1}$ If adequate echocardiographic findings cannot be observed secondary to artifacts, an alternative imaging tool should be considered. In the present case, we describe a patient with infective endocarditis that could not be diagnosed using transthoracic echocardiography (TTE) and transesophageal echocardiography (TEE).

Case Report. A 35-year-old Japanese man had been suffering from high fever $\left(38-39^{\circ} \mathrm{C}\right)$ for more than 14 days. He had a significant medical history of tetralogy of Fallot. When the patient was 28 years, he underwent surgery to repair the tetralogy of Fallot and pulmonary atresia. A conduit containing a mechanical prosthetic valve was inserted at that time to connect the right ventricle and the main pulmonary artery, bypassing the right ventricular outflow tract (RVOT).

On his physical examination, a grade III/VI systolic murmur was audible at the right sternal border. It was noticed that many of his teeth were decayed. Abnormal findings were not observed in the blood and urine tests, except for an elevated white blood cell count $(18,300$ cells $\left./ \mathrm{mm}^{3}\right)$, C-reactive protein $(15 \mathrm{mg} / \mathrm{dL})$, and positive blood cultures. Computed tomography (CT) with and without contrast medium of the head, chest, and

From the Division of General Medicine, Center for Community Medicine, ${ }^{\text {a }}$ Division of Cardiovascular Medicine, Department of Medicine, ${ }^{c}$ Jichi Medical University School of Medicine, Tochigi, Japan; Department of General Medicine, ${ }^{\mathrm{b}}$ Toyooka Public Hospital, Hyogo, Japan; and Department of Family and Community Medicine ${ }^{\mathrm{d}}$ Kobe University Graduate School of Medicine, Hyogo, Japan.

Reprint requests: Tsuneaki Kenzaka, MD, Division of General Medicine, Center for Community Medicine, Jichi Medical University School of Medicine, Tochigi, Japan; smile.kenzaka@jichi.ac.jp.

J Nucl Cardiol 2011;18:741-3.

$1071-3581 / \$ 34.00$

Copyright (C) 2011 The Author(s). This article is published with open access at Springerlink.com.

doi:10.1007/s12350-011-9376-x abdomen did not reveal any cause of his fever. Because of the features of his present illness and his history, infective endocarditis was strongly suspected.

On TTE and TEE, there were no visual findings to clearly show infective endocarditis. Because of artifacts, the pulmonic valve could not be fully visualized. Streptococcus viridans was detected in all five sets of blood cultures (three sets on admission, one set after 12 hours, and the last set at 24 hours after his hospital stay). Because infective endocarditis was suspected, a decision had to be made regarding the need for a surgical procedure (valve repair). For making a decision, it was necessary to identify infectious focal areas and size them. Thus, cardiac 64-slice CT was performed to confirm the presence of vegetations on the valves. We noticed a low-density area in the valves (Figure 1). However, we could not fully differentiate this area from a blurry artifact image in the artificial blood vessel site of the RVOT. As an alternative, a PET scanning was performed. He fasted for 6 hours before the PET procedure. Fifteen minutes before ${ }^{18} \mathrm{~F}$-fluorodeoxyglucose (FDG) injection, unfractionated heparin $(50 \mathrm{IU} / \mathrm{kg}$ ) was preadministered intravenously in an attempt to reduce physiologic ${ }^{18}$ F-FDG uptake by the myocardium. ${ }^{2}$ One hour after injection of $4 \mathrm{MBq} / \mathrm{kg}{ }^{18} \mathrm{~F}-\mathrm{FDG}$, the PET scan was acquired. This scan clearly revealed the localized accumulation of FDG in the artificial blood vessel site of the RVOT (Figure 2).

This patient was treated according to the infective endocarditis therapeutic strategy proposed by the American Heart Association. ${ }^{1}$ The accumulation of FDG in the part of the artificial blood vessel seen in the first PET scan had disappeared in the second scan. The second scan was obtained after the 6-week treatment period (Figure 3). The second PET scan was performed according to the same protocol as the first scan except for heparin administration. The patient recovered with the treatment regimen. On follow-up, he has been in good health for more than a year.

Discussion. The sensitivity and specificity of TEE in infective endocarditis are reported to be $87 \%$ $100 \%$ and $91 \%-100 \%$, respectively. ${ }^{3}$ The utility of multislice CT in the diagnosis of infective endocarditis 


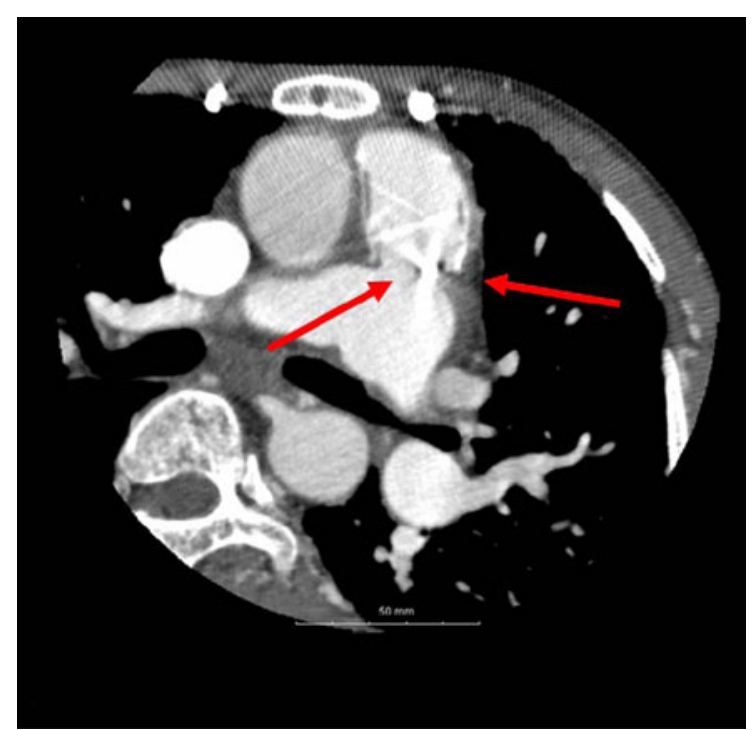

Figure 1. Multislice CT scan images: The images show a low-density area in the pulmonic valve (red arrows). has been reported in recent years. According to those reports, the sensitivity and specificity of multislice CT relative to TEE are $97 \%$ and $87 \%$, respectively. ${ }^{4}$ Even though the present case was evaluated by multislice CT, it was difficult for us to differentiate the verrucous vegetations from the blur caused by artifacts. Recently, it was stated that once the accumulation of FDG is noted on a PET scan in a suspected area, infective endocarditis can be diagnosed using this technique. ${ }^{5,6}$ Alternative radionuclide imaging tests for the diagnosis of infective endocarditis are ${ }^{67} \mathrm{Ga}$ scintigraphy ${ }^{7}$ and ${ }^{99 \mathrm{~m}} \mathrm{Tc}$-mononuclear leukocyte scintigraphy. ${ }^{8}$ As compared with PET, Gallium scintigraphy is time-consuming, and often images are taken over several days. The isotope also has unfavorable physical characteristics for gamma camera imaging. ${ }^{9}{ }^{99 \mathrm{~m}}$ Tc-mononuclear leukocyte scintigraphy could not be performed because of a concern in making in-house labelled leukocytes. Therefore, PET scanning was performed. In the present case, PET scanning detected FDG accumulation in a part of a prosthetic

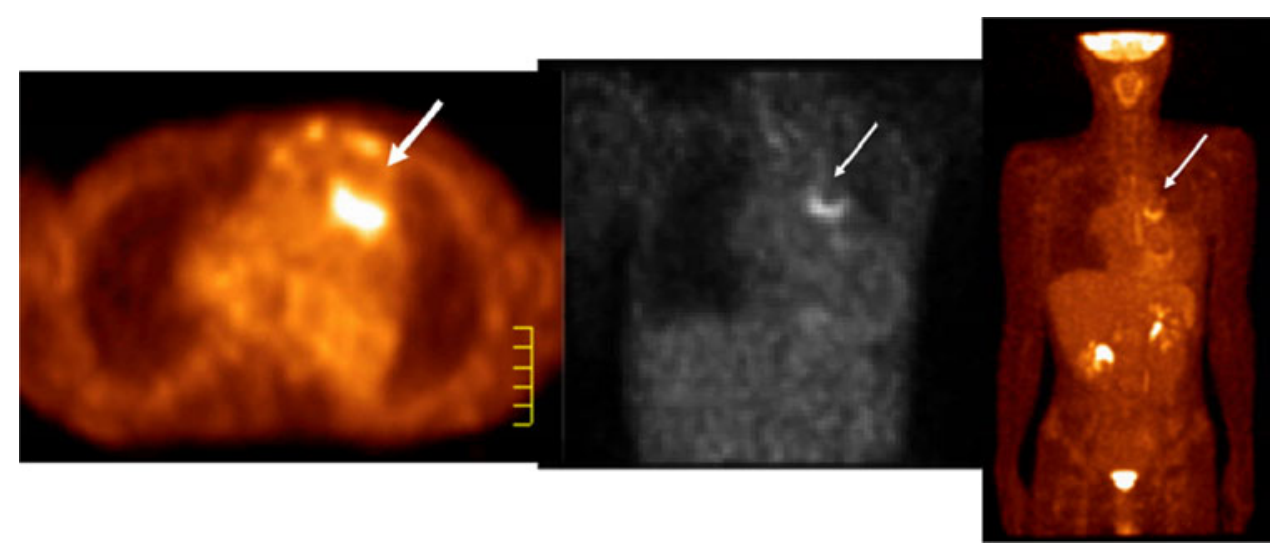

Figure 2. Positron emission tomography (PET) scan images: The images show a focal area of increased FDG uptake in the artificial blood vessel site of the right ventricular outflow tract (white arrows).
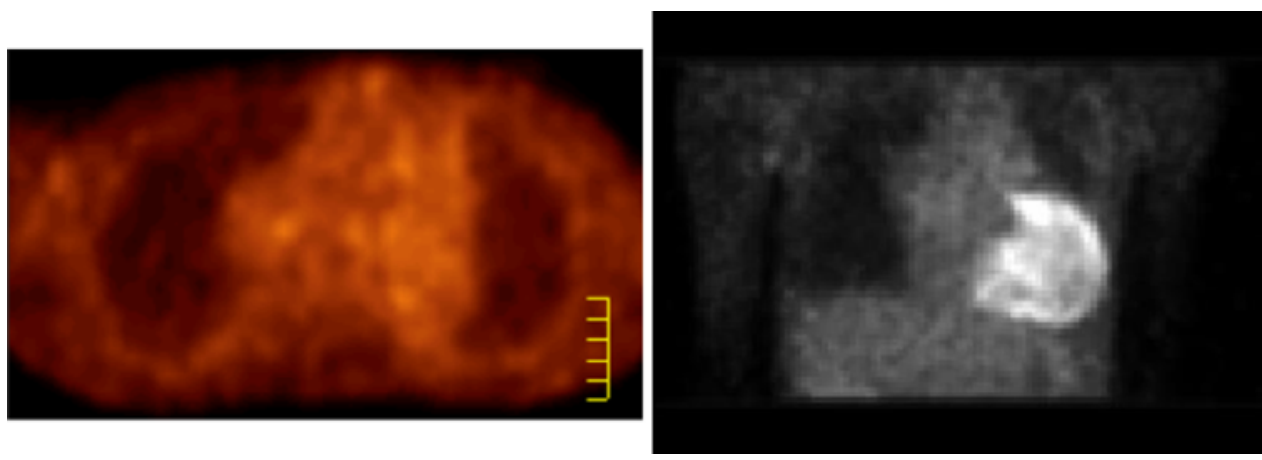

Figure 3. Positron emission tomography (PET) scan images: FDG uptake disappeared after treatment. 
blood vessel of the RVOT. Moreover, this accumulation disappeared after the treatment. PET scan verified that the original FDG accumulation represented localized infection.

It should keep in mind that focal FDG uptake is not specific for the diagnosis of endocarditis. FDG accumulation has been reported with cardiac tumors, ${ }^{10,11}$ active cardiac sarcoidosis, ${ }^{2}$ and even in atheromatous plaques with active inflammation. ${ }^{12}$

In conclusion, we report a case of infective endocarditis that could not be diagnosed using TTE/TEE and multislice CT. PET scanning was shown to be very useful not only for the diagnosis of localized infection in a conduit but also for following the therapeutic response to treatment of infective endocarditis.

\section{Open Access}

This article is distributed under the terms of the Creative Commons Attribution Noncommercial License which permits any noncommercial use, distribution, and reproduction in any medium, provided the original author(s) and source are credited.

\section{References}

1. Baddour LM, Wilson WR, Bayer AS, Fowler VG Jr, Bolger AF, Levison ME, et al. Infective endocarditis: Diagnosis, antimicrobial therapy, and management of complications: A statement for healthcare professionals from the Committee on Rheumatic Fever, Endocarditis, and Kawasaki Disease, Council on Cardiovascular Disease in the Young, and the Councils on Clinical Cardiology, Stroke, and Cardiovascular Surgery and Anesthesia, American
Heart Association: endorsed by the Infectious Diseases Society of America. Circulation 2005;111:e394-434.

2. Nishiyama Y, Yamamoto Y, Fukunaga K, Takinami H, Iwado Y, Satoh K, et al. Comparative evaluation of 18F-FDG PET and $67 \mathrm{Ga}$ scintigraphy in patients with sarcoidosis. J Nucl Med 2006; 47:1571-6.

3. Evangelista A, Gonzalez-Alujas MT. Echocardiography in infective endocarditis. Heart 2004;90:614-7.

4. Feuchtner GM, Stolzmann P, Dichtl W, Schertler T, Bonatti J, Scheffel H, et al. Multislice computed tomography in infective endocarditis: comparison with transesophageal echocardiography and intraoperative findings. J Am Coll Cardiol 2009;53:436-44.

5. Millar BC, Prendergast BD, Alavi A, Moore JE. Positron emission tomography (PET): A new tool in the diagnosis of endocarditis. Heart 2009;95:332-3.

6. Vind SH, Hess S. Possible role of PET/CT in infective endocarditis. J Nucl Cardiol 2010;17:516-9.

7. Thomson LE, Goodman MP, Naqvi TZ, Feldman R, Buchbinder NA, Waxman A, et al. Aortic root infection in a prosthetic valve demonstrated by gallium-67 citrate SPECT. Clin Nucl Med 2005;30:265-8.

8. Gutfilen B, Lopes de Souza SA, Martins FP, Cardoso LR, Pinheiro Pessoa MC, Fonseca LM. Use of ${ }^{99 \mathrm{~m}} \mathrm{Tc}$-mononuclear leukocyte scintigraphy in nosocomial fever. Acta Radiol 2006; 47:699-704.

9. Hughes DK. Nuclear medicine and infection detection: the relative effectiveness of imaging with ${ }^{111} \mathrm{In}$-oxine-, ${ }^{99 \mathrm{~m}} \mathrm{Tc}-\mathrm{HMPAO}$, and ${ }^{99 \mathrm{~m}} \mathrm{Tc}$-stannous fluoride colloid-labeled leukocytes and with ${ }^{67} \mathrm{Ga}-$ citrate. J Nucl Cardiol Technol 2003;31:196-201.

10. Martinez-Rodriguez I, Banzo I, Quirce R, Jimenez-Bonilla JF, Medina-Quiroz P, Rubio-Vassallo AS, et al. F-18 FDG PET/CT uptake by a cardiac hemangioma. Clin Nucl Med 2010;35:330-1.

11. Makis W, Novales-Diaz JA, Lisbona R. Cardiac T-cell lymphoma imaged with F-18 FDG PET-CT and correlative imaging. Clin Nucl Med 2010;35:332-4.

12. Hiari N, Rudd JH. FDG PET imaging and cardiovascular inflammation. Curr Cardiol Rep 2011;13:43-8. 